\title{
Original
}

\section{Structural and Mechanical Properties of the Transparent Dentin Region in the Tooth Root}

\author{
Manabu TABO, Toshiko InOUE*, Makoto SAITO, \\ Fumio Nishimura and Takashi MiYAZAKI
}

\begin{abstract}
Dentin is altered in an age-related manner, such that transparent dentin in the tooth root forms during the aging process. Notably, fractures are more likely to occur in aged teeth. However, few studies have examined the mechanical properties of aged dentin. The purpose of this study was to investigate the structural and mechanical properties of the transparent dentin region, by analysis of the mineral density, using micro-computed tomography (micro-CT), the tensile strength, and nanohardness to understand tooth fracture. As experimental samples, 10 caries-free, human molars were extracted from patients aged $>50$ years because of periodontal disease. As controls, bovine lower central incisors were extracted immediately after sacrifice from animals estimated at 2-2.5 years of age. Mineral density was quantified with X-ray micro-CT analysis of transparent human dentin and normal bovine dentin. Specimens were prepared from each tooth (human and bovine) and subjected to tensile strength testing; maximum load was used to calculate strength, and mean tensile strength of the specimens was calculated in each group. Finally, the nanohardness and Young's modulus of intertubular dentin of the upper and apex root regions were investigated. All results were compared by paired Student's t-test $(\alpha=0.05)$. The mineral density of transparent dentin was significantly greater than that of normal dentin. Importantly, the tensile strength of the transparent regions was significantly lower than that of the normal regions, whereas the nanohardness and Young's modulus of transparent intertubular dentin were significantly greater than those of normal intertubular dentin. Fractures of the teeth remain a major problem in clinical practice. Notably, transparent dentin is weaker than normal opaque dentin, which may explain the occurrence of apex root dentin fractures in aged individuals.
\end{abstract}

Key words : tooth, root, dentin, transparent dentin, mechanical properties

\section{Introduction}

Teeth play critical functional roles in the body, and assessing their mechanical properties is important to understanding such roles. Tooth fracture is a major problem in dentistry and the most common cause of tooth loss. Teeth are composed of a crown and one or more roots, 
with dentin the most abundant mineralized tissue by both weight and volume across the tooth structures ${ }^{1)}$. Thus, the mechanical properties of dentin provide useful information regarding tooth fracture.

Dentin is a hydrated biological composite comprising inorganic hydroxyapatite (HAP) crystallites $(5 \mathrm{~nm}$ thickness, $70 \%$ by weight), organic type I collagen $(50-100 \mathrm{~nm}$ diameter, $18 \%$ by weight $)$, and water $(12 \% \text { by weight })^{2)}$. Dentinal tubules run continuously from the dentinoenamel junction (DEJ) to the pulp in the crown, and from the cementoenamel junction (CEJ) to the pulp canal in the root. The tubule lumina are also surrounded by a collar of hypermineralized peritubular dentin, with the substance between the dentinal tubules known as intertubular $\operatorname{dentin}^{3,4)}$.

Dentin also undergoes age-related changes, most notably apparent by the formation of transparent dentin in the tooth root that typically starts at the apical end of the root and often extends into the coronal dentin ${ }^{5)}$. Aged teeth are also more susceptible to fractures, increasing the importance of the age-related alterations in dentin. Unfortunately, studies regarding the mechanical properties of aged dentin are scarce. There is some evidence that both the fracture toughness of transparent dentin and fatigue strength of aged dentin are lower than normal in young dentin ${ }^{6}$, and tensile or fatigue strength testing has long been used as a method to test tooth strength ${ }^{7-10)}$. Importantly, there are scarce data regarding the tensile strength of transparent dentin in aged human teeth.

Dentin transparency is a commonly observed pathology in aged teeth; however, it remains unclear whether corresponding alterations are apparent in the intertubular dentin matrix. Nanohardness as well as Young's modulus ${ }^{11)}$ enable investigation of tooth structure in the dentin region. Additionally, transparent dentin represents increased mineralization of the dentin matrix ${ }^{6}$. MicroCT is a useful method to observe and clear mineral density ${ }^{12-15)}$; thus we aimed herein to investigate the structural and mechanical properties of the transparent dentin region (tensile strength, nanohardness, and micro-CT) to better understand the nature and timing of tooth fracture.

\section{Materials and methods}

\section{Materials}

Ten extracted human premolars and molars were obtained from patients aged $>50$ years (5273 years) following the receipt of informed consent. Importantly, the molars were extracted because of periodontal disease. The protocol of this study was approved by the Ethics Committee of Showa University School of Dentistry (2008-26).

Ten bovine lower central incisors were also extracted immediately after sacrifice from animals estimated to be 2-2.5 years old, based on their dental age. Human and bovine teeth were stored in Hank's balanced saline solution (HBSS) at $4^{\circ} \mathrm{C}$, and were used within 1 month of extraction, in accordance with previous reports ${ }^{7-9)}$.

\section{Measurement of mineral density}

We quantified the mineral density of dentin in the human (Group 1) (transparent region) 


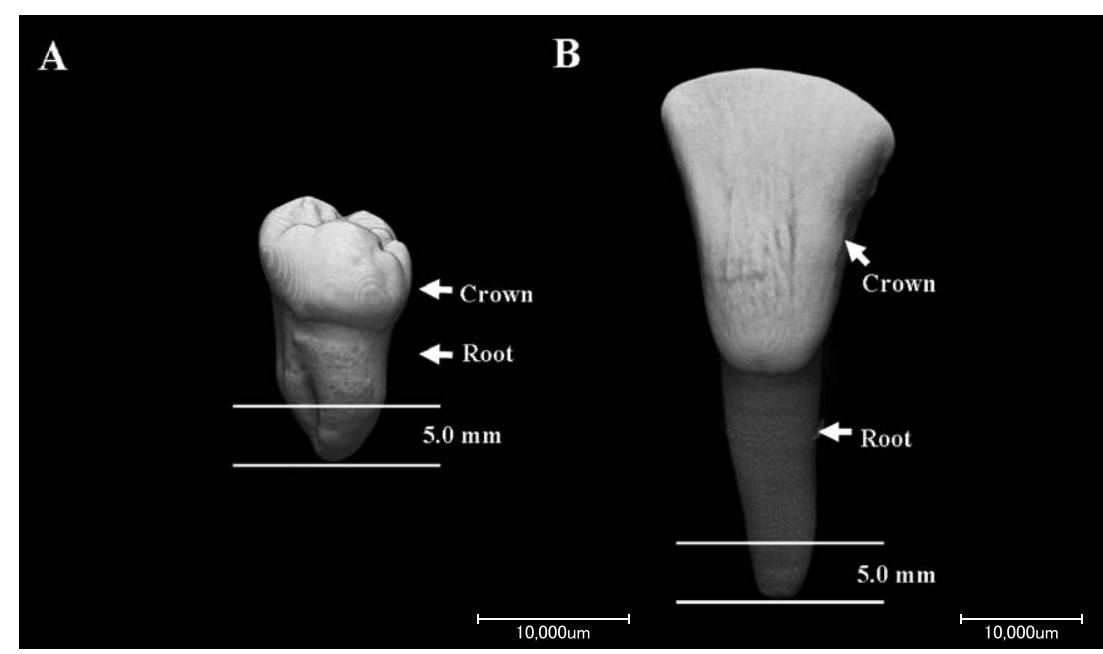

Fig. 1. Schematic representation of the region of interest (ROI) for each tooth. (A) human tooth and (B) bovine tooth.

Table 1. Experimental groups for mineral density measurements and tensile test

\begin{tabular}{cl}
\hline Groups & Tooth Type \\
\hline 1 & Human teeth \\
2 & Bovine teeth \\
\hline
\end{tabular}

and bovine (Group 2) (normal region) teeth using micro-CT analysis, as shown in Figure 1 and Table 1. The region of interest (ROI) was from the apex to $5.0 \mathrm{~mm}$ coronal to the crown area in all teeth. Specimens were scanned at $50 \mathrm{keV}$ and $80 \mu \mathrm{A}$ using an X-ray micro-CT system (SMX-90, Shimadzu, Kyoto, Japan), with rotation through $360^{\circ}$ in steps of $0.2^{\circ}$. An aluminum filter was placed in front of the detector to remove low-energy X-rays, and 3D software (TRI/3D-BON, Ratoc System Engineering Company Limited, Tokyo, Japan) was used to visualize and analyze the mineral density, in accordance with a previous report ${ }^{12}$. Mean mineral density values were compared by paired Student's t-test; $P<0.05$ was considered significant.

\section{Preparation of specimens}

All teeth were sectioned midway along the distal portions of the buccolingual plane along the axis of the tooth using a diamond saw (Isomet, Beuhler, Lake Bluff, IL, USA) (Figure 2). Figure 3 depicts a control human tooth aged appropriately. One dentin slab, approximately $1-\mathrm{mm}$ thick, was cut from each tooth, and a rectangular block, as shown in Figure $2(3.0 \times 3.0 \times$ $1.0 \mathrm{~mm}$ ), was obtained from the root apex region. Dumbbell-shaped specimens (central portion; $1.0 \times 1.0 \times 1.5 \mathrm{~mm}$ ) from each slab were then prepared using a custom-made profiling machine and stored in HBSS to maintain moisture ${ }^{7-9)}$. 


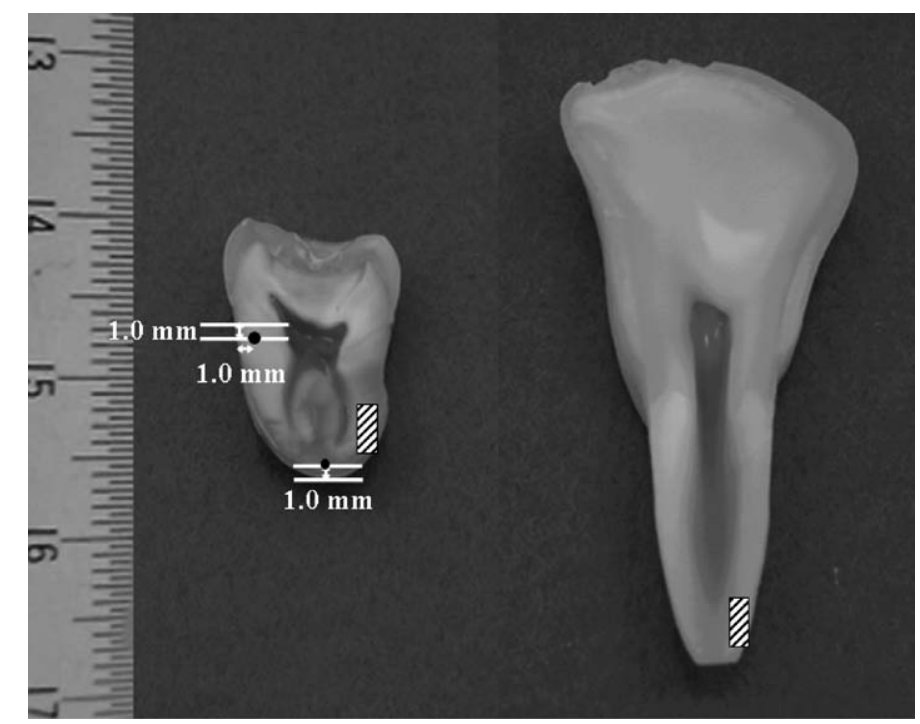

Fig. 2. The locations of tensile test specimens from a human molar tooth (left image) and a bovine incisor tooth (right image). In human teeth (left), the two areas of nanohardness and Young's modulus measurements were designated as upper root and apex root.

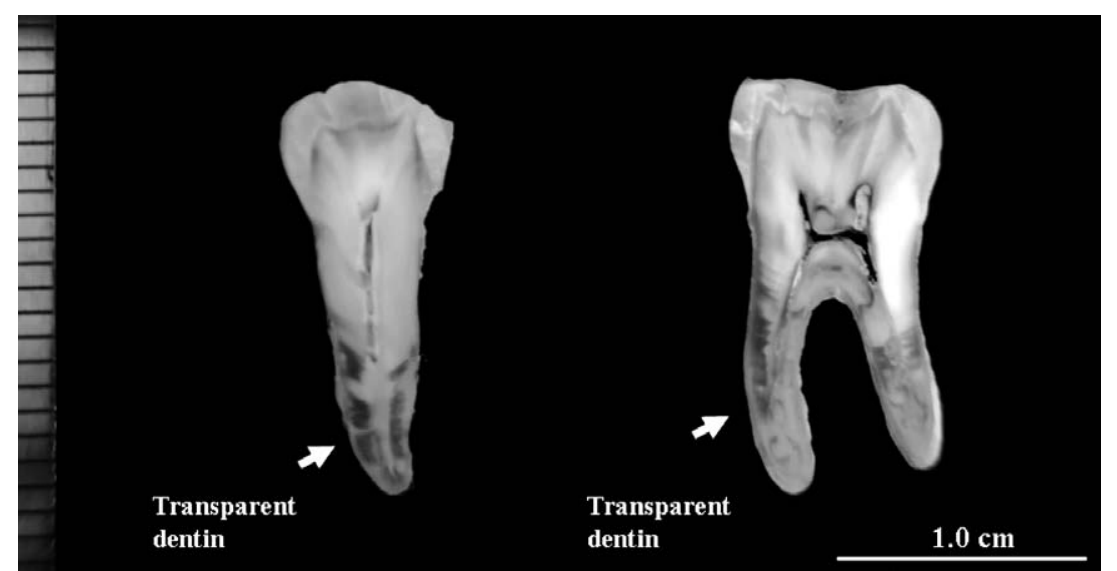

Fig. 3. Typical transparent dentin in human teeth

\section{Tensile strength test}

Specimens were placed on a universal testing machine (EKO Instruments Co., Ltd., Tokyo, Japan) immediately after preparation ${ }^{7-9)}$ and stored in $37 \pm 0.5^{\circ} \mathrm{C}$ HBSS during the tensile strength test. The maximum load was used to calculate strength. Ten specimens in each experimental group were tested, and mean tensile strength was calculated in each group (Figure 4). The results were compared by paired Student's t-test $(P<0.05)$. 



Fig. 4. Preparation for tensile test for specimen

Table 2. Experimental groups for nanohardness and Young's modulus measurements

\begin{tabular}{ccc}
\hline Groups & Tooth Type & Location \\
\hline 3 & Human teeth & Upper root \\
4 & Human teeth & Apex root \\
\hline
\end{tabular}

\section{Nanohardness and Young's modulus test}

After tensile testing of the human teeth, the remaining specimens were used for nanohardness and Young's modulus measurements of intertubular dentin of the upper and apex root regions, as indicated in Figure 2 and Table 2. Specimens were trimmed with indentation positions as follows:

Group 3: upper root region in human teeth, $1.0 \mathrm{~mm}$ midway from the outer surface and $1.0 \mathrm{~mm}$ below the CEJ.

Group 4: apex root region in human teeth, $1.0 \mathrm{~mm}$ midway from the outer surface and $1.0 \mathrm{~mm}$ above the apex.

A hardness tester (DUH-W201, Shimadzu, Kyoto, Japan) attached to a Berkovich indenter and a three-sided pyramid diamond probe was used, with the specimen surface orientated parallel to the stage. Nanohardness and Young's modulus were measured using a force up to $5 \mathrm{mN}$ to create a superficial indent on the specimen surface in accordance with a previous report ${ }^{11)}$.

\section{Results}

\section{Mineral density of transparent dentin region}

The mineral densities of each group are shown in Figure 5, depicted as mean and standard deviation. The mineral density of transparent dentin within the ROI in human teeth was 1732.6 


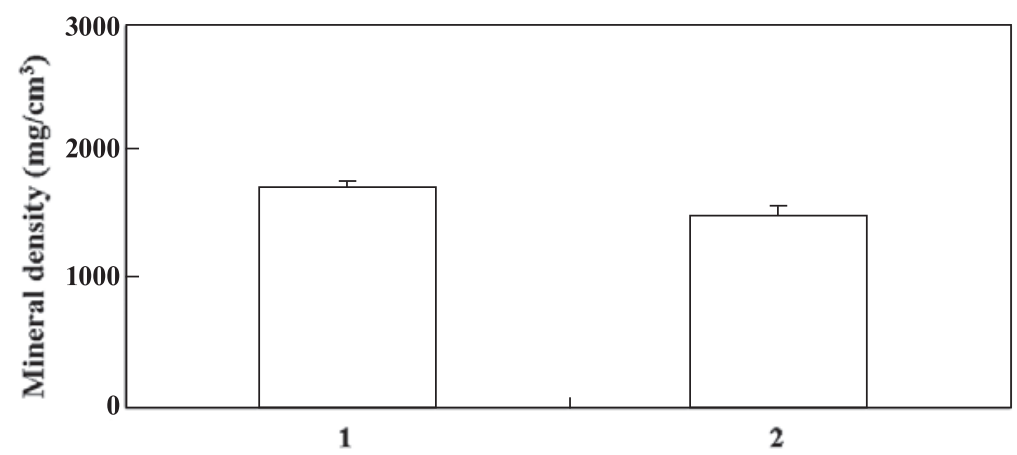

Fig. 5. Mineral density of human (1) and bovine teeth (2)
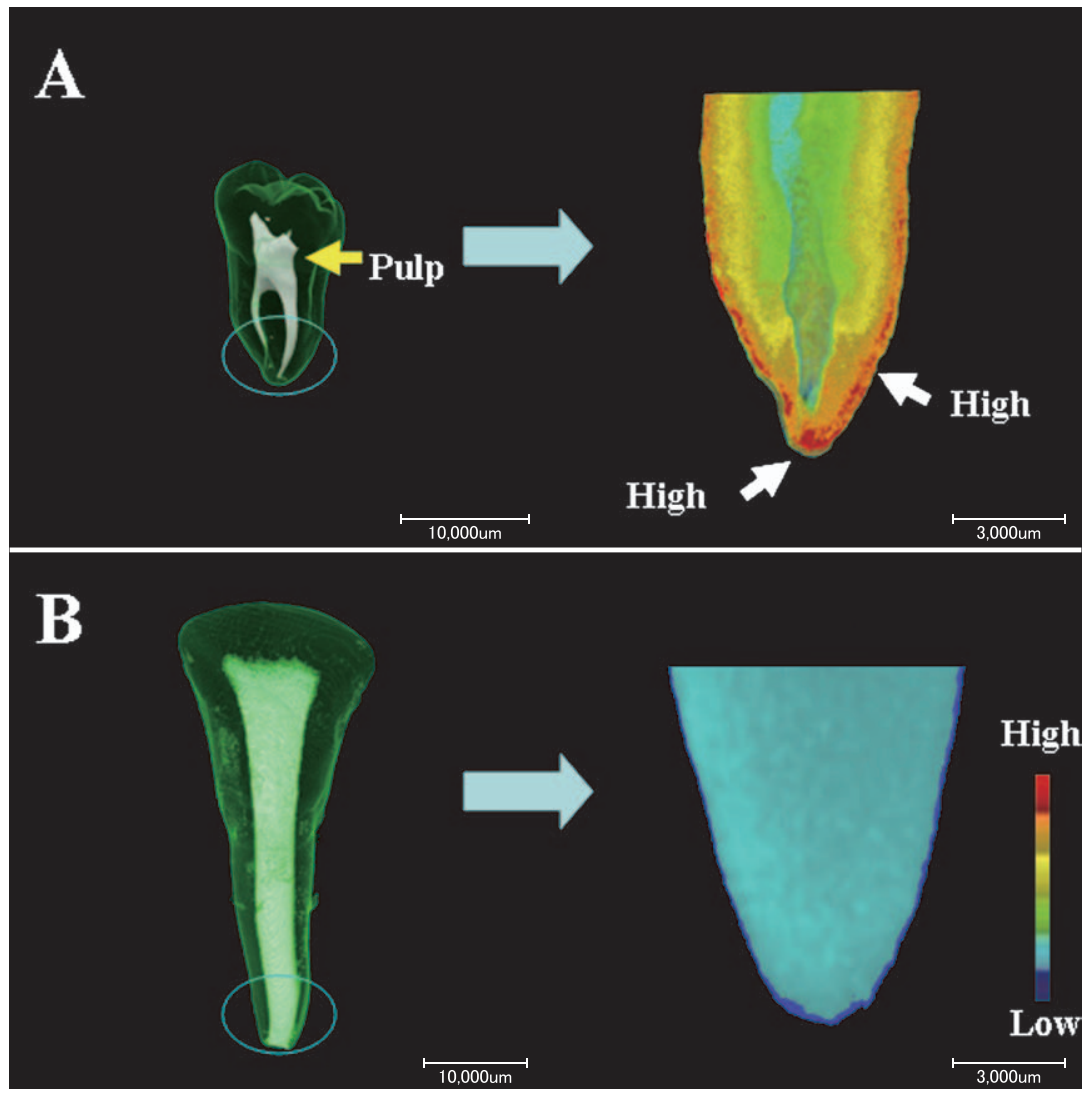

Fig. 6. Pseudo-color three-dimensional images of teeth generated from micro-CT data of human (A) and bovine teeth (B)

$\pm 45.3 \mathrm{mg} / \mathrm{cm}^{3}$, whereas that of normal dentin within the ROI (bovine teeth) was $1388.7 \pm 65.8$ $\mathrm{mg} / \mathrm{cm}^{3}$. The mineral density of transparent dentin (human teeth) was significantly greater than that of normal dentin (bovine teeth) as shown in Figure 6.

Tensile strength of transparent and normal regions in the tooth root

The tensile strengths of the two groups are shown in Figure 7. The mean tensile strength was 


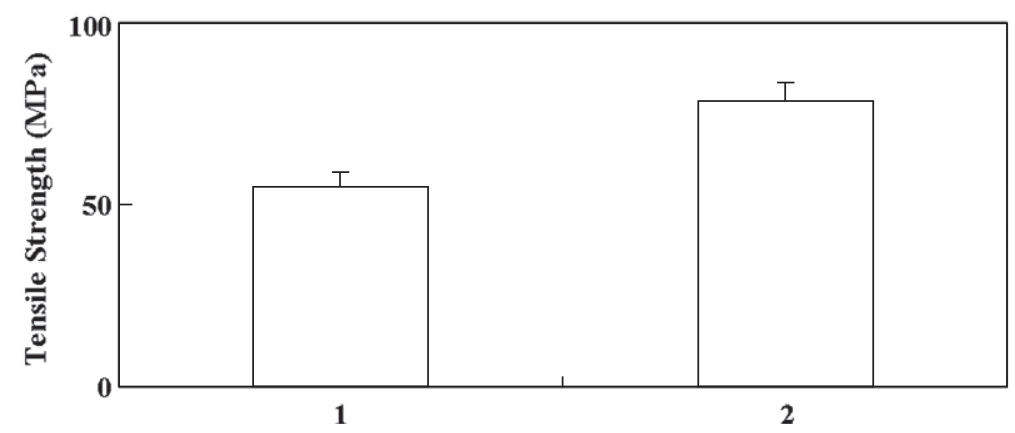

Fig. 7. Tensile strength of human (1) and bovine teeth (2)

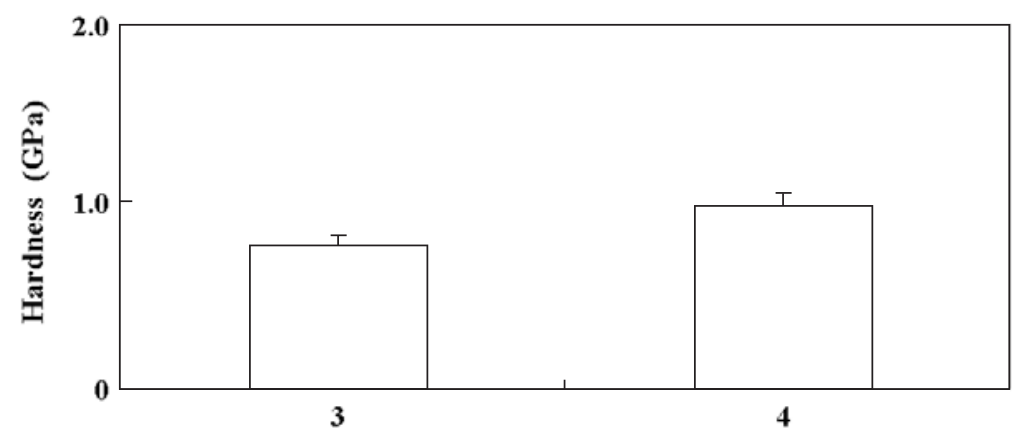

Fig. 8. Nanohardness of the upper (3) and apex (4) roots in human teeth

significantly lower in the transparent regions at $56.4 \pm 3.3 \mathrm{MPa}$ compared to the normal regions at $78.6 \pm 4.8 \mathrm{MPa}(P<0.05)$.

Nanohardness and Young's modulus of transparent and normal dentin

The measured nanohardness and Young's modulus are indicated in Figures 8 and Figure 9, respectively, with nanoindentation test results shown for transparent (apex root) and normal intertubular dentin (upper root) at two locations of each tooth. Mean nanohardness and Young's modulus were significantly different between the dentin types $(P<0.05)$. The nanohardness of transparent dentin was $1.0 \pm 0.05 \mathrm{GPa}$ and that of normal dentin was $0.8 \pm 0.02 \mathrm{GPa}$. Additionally, the Young's modulus of transparent dentin was $25.3 \pm 2.1 \mathrm{GPa}$ and that of normal dentin was $22.4 \pm 1.1 \mathrm{GPa}$. The nanohardness and Young's modulus of transparent intertubular dentin were thus greater than those of normal intertubular dentin.

\section{Discussion}

These results confirm that transparent dentin has a lower tensile strength than normal dentin, and that the altered dentin showed increased values for mineral density, nanohardness and Young's modulus.

We used teeth from humans aged over 50 years based on reports of the point of aging in 


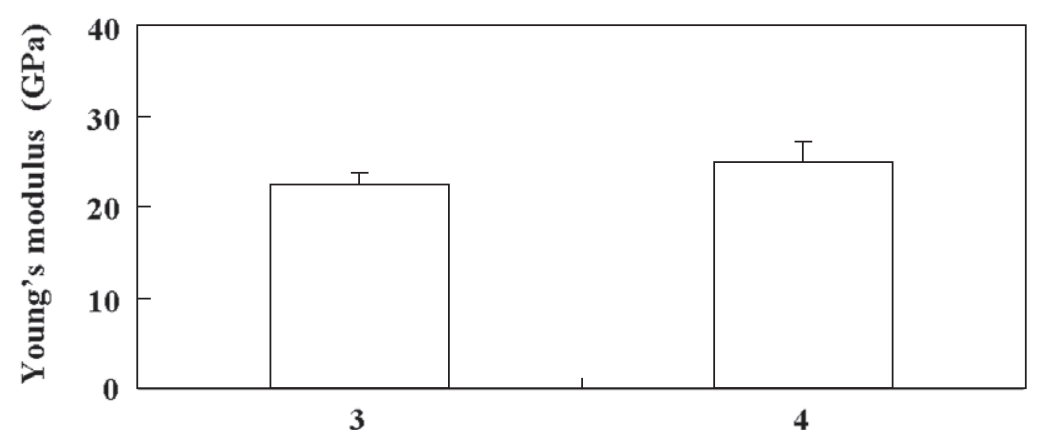

Fig. 9. Young's modulus of the upper (3) and apex (4) roots in human teeth

terms of teeth ${ }^{16)}$. In this study, we used bovine teeth to compare normal dentin with transparent dentin because bovine teeth are a good model for human teeth in dental research ${ }^{17)}$. Furthermore, it is easier to collect the same types of bovine teeth, of the same age, in sufficient numbers, while individual history and hereditary characteristics vary much less among bovine teeth than among humans because of selective breeding of cattle in Japan ${ }^{17)}$. Additionally, apical translucency was confirmed in the youngest age group (16 years) in human teeth ${ }^{18)}$, whereas in cattle of 2.0-2.5 years of age, permanent teeth erupt soon after deciduous teeth and thus, there is no effect of aging. The strength of bovine dentin does not apply to humans, and anisotropic tensile strength of crown dentin differs between bovine and human teeth because of incremental lines ${ }^{7)}$. Nevertheless, bovine dentin is a well recognized substitute for human dentin in adhesive dentistry ${ }^{19)}$.

Tensile strength of dentin varies according to the location and orientation of dentinal tubules in the tooth ${ }^{20-22)}$, with the tensile strengths of radicular dentin significantly greater than those of coronal dentin along the tooth axis. Thus, the tensile strength of dentin was strongest in bovine teeth in this study, although the tensile strength of transparent human dentin was lower than that of bovine dentin, possibly reflecting an effect due to the aged intertubular dentin (Figure 8). Our study also confirmed that transparent dentin is harder than normal dentin. According to a previous report, coronal intertubular dentin is harder than radicular intertubular dentin ${ }^{11)}$. Further, the tensile strength of coronal dentin is lower than that of radicular dentin ${ }^{20)}$. The harder the intertubular dentin, the lower the tensile strength of dentin. Thus, the tensile strength of transparent dentin is lower than that of normal dentin.

In this study, transparent intertubular dentin was harder than normal dentin, possibly indicating a lower tensile strength; however, the nanohardness and Young's modulus of normal human dentin $(0.8 \mathrm{GPa}$ and $22.4 \mathrm{GPa})$ and transparent human dentin (1.0 GPa and 25.3 GPa) are reportedly greater than those of bovine radicular teeth $(0.6 \mathrm{GPa} \text { and } 20.8 \mathrm{GPa})^{11}$. Notably, lower tissue mineral content correlates with lower modulus and nanohardness. Additionally, in this study, we examined the hardness and Young's modulus of the intertubular dentin, but not those of peritubular dentin, because intertubular dentin dominates the strength value of dentin ${ }^{11)}$. Furthermore, in aged dentin, the transparent dentin (apex root) and normal dentin (opaque upper root) 
differ with respect to nanohardness and Young's modulus. The mechanical properties of dentin thus become structurally localized with aging. Finally, the fracture toughness of aged dentin, as measured in a previous report, was found to be lowered by roughly $20 \%{ }^{23)}$. In our study, the tensile strength of aged dentin was lower than that of normal dentin, which is agreement with the previous study.

\section{Conclusion}

Fractures of the teeth remain a major problem in clinical practice. An understanding of structural tooth strength is therefore important to improve our understanding of tooth fractures. We found herein that transparent dentin is weaker than normal opaque dentin, possibly explaining the occurrence of apex root dentin fractures in aged individuals.

\section{Acknowledgements}

This work supported by a Grant-in-Aid for Challenging Exploratory Research (no. 15k15715) and a Grant-in-Aid for Scientific Research (C) (no. 25463021) from the Japan Society for the Promotion of Science.

\section{Conflict of interest disclosure}

There are no conflicts to disclose.

\section{References}

1) Inoue $T$, Yamamoto $M$, Debari $K$, et al. Chemical analysis of coronal and radicular dentin using Fourier transform infrared spectroscopy. Dent Med Res. 2009;29:134-138.

2) Lussi A. Dental erosion from diagnosis to therapy. Basel: Karger; 2006.

3) Nanci A. Ten Cate's oral histology: development, structure and function. 7th ed. St. Louis: Mosby; 2008.

4) Inoue $\mathrm{T}$, Debari $\mathrm{K}$, Kou K, et al. Dentinal tubule densities in human and bovine coronal dentin. Dent Med Res. 2009;29:238-240.

5) Vasiliadis L, Darling AI, Levers BG. The amount and distribution of sclerotic human root dentine. Arch Oral Biol. 1983;28:645-649.

6) Kinney JH, Nalla RK, Pople JA, et al. Age-related transparent root dentin: mineral concentration, crystallite size, and mechanical properties. Biomaterials. 2005;26:3363-3376.

7) Inoue $\mathrm{T}$, Saito $\mathrm{M}$, Yamamoto $\mathrm{M}$, et al. Relation between incremental lines and tensile strength of coronal dentin. Dent Mater J. 2012;31:541-548.

8) Inoue $\mathrm{T}$, Nishimura $\mathrm{F}$, Debari $\mathrm{K}$, et al. Fatigue and tensile properties of radicular dentin substrate. $J$ Biomech. 2011;44:586-592.

9) Inoue T, Miyazaki T, Nishimura F. Tensile strength and durability of bovine dentin. Dent Mater J. 2007;26:348-354.

10) Inoue $\mathrm{T}$, Saito $\mathrm{M}$, Yamamoto $\mathrm{M}$, et al. Fatigue and fracture observations of radicular dentin. Dent Med Res. 2011;31:228-231.

11) Inoue $T$, Saito $M$, Yamamoto $M$, et al. Comparison of nanohardness between coronal and radicular intertubular dentin. Dent Mater J. 2009;28:295-300.

12) Inoue $\mathrm{T}$, Saito M, Yamamoto $\mathrm{M}$, et al. Mineral density of coronal and radicular dentin. Dent Med Res. 2013;33:248-251.

13) Inoue $\mathrm{T}$, Saito $\mathrm{M}$, Nishimura $\mathrm{F}$, et al. Three-dimensional representation of microdontia of the maxillary third molar. 
Clin Case Rep. 2017;5:547-548.

14) Inoue $\mathrm{T}$, Saito $\mathrm{M}$, Nishimura $\mathrm{F}$, et al. Unusual root canal anatomy in a maxillary second molar. Int $J$ Case Rep Images. 2017;8:352-354.

15) Inoue $\mathrm{T}$, Saito $\mathrm{M}$, Nishimura $\mathrm{F}$, et al. Three-dimensional representation of teeth with root dilaceration. Int $J$ Case Rep Images. 2016;7:864-866.

16) Tsai SS, Lin YS, Hwang JS, et al. Vital roles of age and metabolic syndrome-associated risk factors in sex-specific arterial stiffness across nearly lifelong ages: possible implication of menopause and andropause. Atherosclerosis. 2017;258:26-33.

17) Tonami K, Takahashi H. Effects of aging on tensile fatigue strength of bovine dentin. Dent Mater J. 1997;16:156169.

18) M S, G S M, Basandi P, et al. Effect of vitality on translucent dentine-a study. J Int Oral Health. 2013;5:1-7.

19) Nakabayashi N, Watanabe A, Arao T. A tensile test to facilitate identification of defects in dentine bonded specimens. J Dent. 1998;26:379-385.

20) Inoue $\mathrm{T}$, Takahashi $\mathrm{H}$, Nishimura $\mathrm{F}$. Anisotropy of tensile strengths of bovine dentin regarding dentinal tubule orientation and location. Dent Mater J. 2002;21:32-43.

21) Carvalho RM, Fernandes CA, Villanueva R, et al. Tensile strength of human dentin as a function of tubule orientation and density. J Adhes Dent. 2001;3:309-314.

22) Lertchirakarn V, Palamara JE, Messer HH. Anisotropy of tensile strength of root dentin. J Dent Res 2001;80:453456.

23) Kinney JH, Nalla RK, Pople JA, et al. Age-related transparent root dentin: mineral concentration, crystallite size, and mechanical properties. Biomaterials. 2005;26:3363-3376.

[Received August 28, 2018: Accepted October 20, 2018] 